\title{
Akute Lymphangitis
}

\author{
H. S. FüeßI
}

\author{
Das 14-jährige Mädchen wurde am Vortag von \\ einem Insekt am linken Handgelenk gestochen, \\ möglichweise von einer Bremse. Am nächsten \\ Morgen hat sich eine streifenförmige, leicht \\ schmerzhafte Rötung ausgehend von der Einstich- \\ stelle bis in die Ellenbeuge ausgebildet (Abb. 1). \\ Das Mädchen fühlt sich kränklich und hat $38,3^{\circ} \mathrm{C}$ \\ Fieber. Axilläre Lymphknoten sind nicht tastbar. \\ Die Mutter äußert besorgt den Verdacht, dass es \\ sich um eine „Blutvergiftung“ handeln könnte.
}

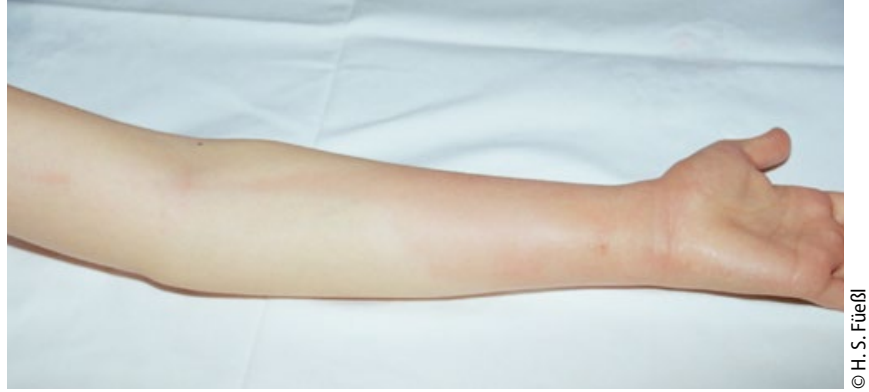

Abb. 1 Nach proximal wandernde Rötung am linken Unterarm; Einstichstelle ulnar am Handgelenk.

\section{Situation und Symptomatik}

Es handelt sich hier um eine sichere Blickdiagnose: Anamnese und klinischer Befund sprechen für eine akute Lymphangitis.

Die akute Lymphangitis wird im Volksmund fälschlicherweise als Blutvergiftung bezeichnet. Charakteristisch ist der bekannte rote Streifen, der „in Richtung Herz" wandert. Es handelt sich aber nicht um eine Sepsis, sondern um eine Entzündung der Lymphbahnen.

Die Erreger der Lymphangitis sind meist Bakterien, v. a. Staphylokokken und Streptokokken. Auch andere Noxen, z. B. Schlangengifte, paravenöse Injektionen von Fremdsubstanzen (Drogenabhängige) oder Medikamente können Ursache einer akuten Lymphangitis sein. Ganz abwegig ist der umgangssprachliche Begriff der Blutvergiftung aber nicht, da sich aus einer Lymphangitis durchaus eine Sepsis entwickeln kann.

Sind die regionären Lymphknoten beteiligt, spricht man von einer Lymphadenitis.

Die Lymphangitis kann ihren Ausgang von einer vorher bestehenden Lokalinfektion, z. B. einem Abszess, nehmen. Im vorliegenden Fall müssen aber Erreger oder Toxine durch den Insektenstich in den Körper gelangt sein. Auch wenn bei dem Kind (noch) keine Beteiligung der regionären Lymphknoten festgestellt werden konnte, spricht das leichte Fieber für eine Allgemeinreaktion auf die lokale Noxe.

\section{Sofortdiagnostik}

Das klinische Bild ist so typisch, dass in diesem Fall keine weiterführenden Untersuchungen für die Diagnosestellung erforderlich sind. Man könnte ein Blutbild, eine BKS oder ein CRP untersuchen, doch sind diese Ergebnisse für die therapeutische Entscheidung nicht wesentlich.

\section{Therapie}

Welche Therapie man im vorliegenden Fall wählt, ist nicht anhand von evidenzbasierten Daten zu entscheiden. Die rasche Ausdehnung des Lokalbefundes, das leichte Fieber und das allgemeine Krankheitsgefühl sprechen für eine antibiotische Therapie, z. B. mit Amoxicillin oder einem Cephalosporin. Allerdings sind auch relativ harmlose Verläufe einer Lymphangitis nach Insektenstich bekannt.

Woran noch denken, was noch tun? Der Verlauf war in diesem Fall glücklich, doch muss das nicht immer so sein. Es

\section{Kasuistik}

\section{WIE GING ES WEITER?}

Nach eingehender Beratung mit der Mutter, die einer Antibiotikatherapie eher ablehnend gegenübersteht, beschließt man, nur lokal mit einem Alkoholverband und einer Diclofenachaltigen Salbe zu behandeln. Allerdings wird das Mädchen für den nächsten Tag zu einer Kontrolluntersuchung einbestellt. Tatsächlich ist die Rötung rückläufig, die Schmerzen haben nachgelassen und es ist kein Fieber mehr vorhanden.

wäre durchaus möglich gewesen, dass es zu einer Sepsis oder einer Abszedierung kommt. Eine engmaschige Überwachung ist sicher angezeigt. Ein kluger Arzt bezieht gerade bei Kindern die Eltern immer in seine Überlegungen und das Vorgehen mit ein.

\footnotetext{
$\rightarrow$ Anschrift des Verfassers:

Prof. Dr. med. Hermann S. FüeßI Internist und Gastroenterologe Privatpraxis für Integrative Innere Medizin Renatastr. 30 D-80639 München

E-Mail: hsfuessl@t-online.de
} 